\title{
Rats depend on habit memory for discrimination learning and retention
}

\author{
Nicola J. Broadbent, ${ }^{1}$ Larry R. Squire, 1,2,3,4 and Robert E. Clark ${ }^{1,2,5}$ \\ ${ }^{1}$ Department of Psychiatry, University of California, La Jolla, California 92093, USA; ${ }^{2}$ Veterans Affairs Medical Center, San Diego, \\ California 92161, USA; ${ }^{3}$ Department of Neurosciences, University of California, La Jolla, California 92093, USA; ${ }^{4}$ Department of \\ Psychology, University of California, La Jolla 92093, USA
}

\begin{abstract}
We explored the circumstances in which rats engage either declarative memory (and the hippocampus) or habit memory (and the dorsal striatum). Rats with damage to the hippocampus or dorsal striatum were given three different two-choice discrimination tasks (odor, object, and pattern). These tasks differed in the number of trials required for learning $(\sim 10,60$, and 220 trials). Dorsal striatum lesions impaired discrimination performance to a greater extent than hippocampal lesions. Strikingly, performance on the task learned most rapidly (the odor discrimination) was severely impaired by dorsal striatum lesions and entirely spared by hippocampal lesions. These findings suggest that discrimination learning in the rat is primarily supported by the dorsal striatum (and habit memory) and that rats engage a habit-based memory system even for a task that takes only a few trials to acquire. Considered together with related studies of humans and nonhuman primates, the findings suggest that different species will approach the same task in different ways.
\end{abstract}

In the mammalian brain, the hippocampus and the dorsal striatum support fundamentally distinct forms of memory (Squire 1992; Schacter and Tulving 1994; Poldrack and Packard 2003). The hippocampal region (the CA fields, dentate gyrus, and subicular complex) and adjacent cortices are important for declarative memory (Squire and Zola-Morgan 1991), and the dorsal striatum supports habit memory (Mishkin et al. 1984). Declarative memory supports the learning of relationships among items and events and appears especially well suited for rapid, even onetrial learning. The hippocampus and related structures support learning in a variety of memory tasks, including spatial tasks like the water maze (Morris et al. 1982), as well as nonspatial tasks like social transmission of food preference (Bunsey and Eichenbaum 1995) and delayed nonmatching to sample (Clark et al. 2001). In contrast, habit memory is typically acquired gradually over many trials through the establishment of stimulus-response associations (Mishkin et al. 1984; Packard et al. 1989; Bayley et al. 2005). It is rigidly organized such that what is learned is not readily expressed except when the task is presented just as it was during training.

One idea that has seemed helpful in explaining whether tasks will depend on declarative memory (and the hippocampus) or on habit memory (and the dorsal striatum) is that the two memory systems acquire information at different rates. An early review of discrimination learning in nonhuman primates (Squire and Zola-Morgan 1983) found that tasks acquired rapidly were more sensitive to the effects of medial temporal lobe damage than tasks acquired slowly. For example, intact monkeys learned pattern discrimination and concurrent discrimination tasks gradually over hundreds of trials but could learn object discriminations in 10 or 20 trials (Moss et al. 1981; Zola-Morgan and Squire 1984; Teng et al. 2000). Correspondingly, monkeys with hippocampal damage acquired pattern discrimination and concurrent discriminations normally (Zola-Morgan and Squire 1984; Buffalo et al. 1998) but were impaired at learning the easier object

\section{${ }^{5}$ Corresponding author.}

E-mail reclark@ucsd.edu; fax (858) 534.1569.

Article is online at http://www.learnmem.org/cgi/doi/10.1101//m.455607. discriminations (Teng et al. 2000). Conversely, monkeys with damage that included the tail of the caudate were impaired at acquiring pattern discriminations as well as concurrent discrimination tasks (Divac et al. 1967; Teng et al. 2000; Fernandez-Ruiz et al. 2001). Furthermore, when rats were trained in a T-maze, performance during the early trials relied on a hippocampusdependent form of place learning. With extended training, the rats shifted to a dorsal striatum-dependent strategy based on response learning (Packard and McGaugh 1996).

Although this distinction between fast and slow learning has been useful, newer findings suggest that rate of learning cannot fully account for which memory system is used to support learning. For example, in one study performance on an odor discrimination task, which rats learned in fewer than 10 trials, was unaffected by hippocampal lesions (Jonasson et al. 2004). Rats with hippocampal lesions learned the task as rapidly as intact animals and retained the task at normal levels $7 \mathrm{~d}$ later. In this case, the hippocampus was not important, even though the task was acquired rapidly.

One hint about what factors might be important comes from increasing evidence that different species will approach the same task in different ways. Perhaps the clearest example lies in how humans and monkeys approach the standard eight-pair, concurrent discrimination task. In this task, eight pairs of objects are presented five times each day, one pair at a time, and in a mixed order. One object in each pair is always correct, and a choice of the correct object yields a reward. Humans learn the task by memorizing the correct object in each pair, and master the task in one or two sessions. Patients with hippocampal lesions were impaired at acquiring this task (Squire et al. 1988; Hood et al. 1999; Bayley et al. 2005). In sharp contrast, monkeys solved the concurrent discrimination task in several hundred trials and independently of the hippocampus. For monkeys, the concurrent discrimination task depends on the caudate (Teng et al. 2000; Fernandez-Ruiz et al. 2001). If monkeys are more likely than humans to engage habit memory to acquire certain tasks, rats might have this same tendency (or perhaps even a greater tendency) to engage habit memory. Indeed, habit memory is thought to be a phylogenetically early kind of memory, and 
habit memory might be increasingly more dominant than declarative memory as one moves from humans to monkeys to rats.

We have investigated how readily rats rely on habit memory relative to hippocampus-dependent memory. We compared the effects of lesions to the dorsal striatum (caudate and putamen in the rat) (Packard and Knowlton 2002) or hippocampus on acquisition and retention of three discrimination tasks that differed in how rapidly they could be learned. We used a two-choice odor discrimination task that could be learned in $\sim 10$ trials, a twochoice object discrimination task that could be learned in $\sim 60$ trials, and a two-choice pattern discrimination task that could be learned in $\sim 220$ trials, and we used both retrograde and anterograde designs. The findings show that rats rely heavily on a habitbased memory strategy to solve discrimination tasks, regardless of whether the task is learned rapidly or only gradually.

\section{Results}

\section{Histology}

\section{Hippocampal lesions}

Figure 1A shows the smallest (black) and largest (stippled) extent of the hippocampal lesion. All animals sustained extensive damage to all the cell fields of the hippocampus, including the dentate gyrus. The mean percent damage was $86.1 \%$ of total hippocampal volume (range, 66.1\%-99.8\%). In cases where the lesion was not complete at a particular level of the hippocampus, the sparing was typically restricted to the most medial aspect of the dorsal dentate gyrus or the dorsal CA1 cell field, or to the most ventromedial aspect of the ventral hippocampus. All animals (except one) had damage to the subicular complex, and the mean percent damage was $33.5 \%$ (range, 4.7\%-91.2\%). Eleven of the 18 animals also had minor damage to the entorhinal cortex
A

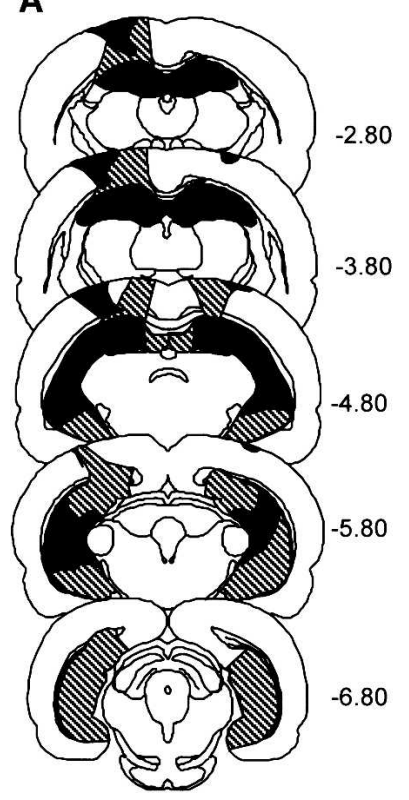

B

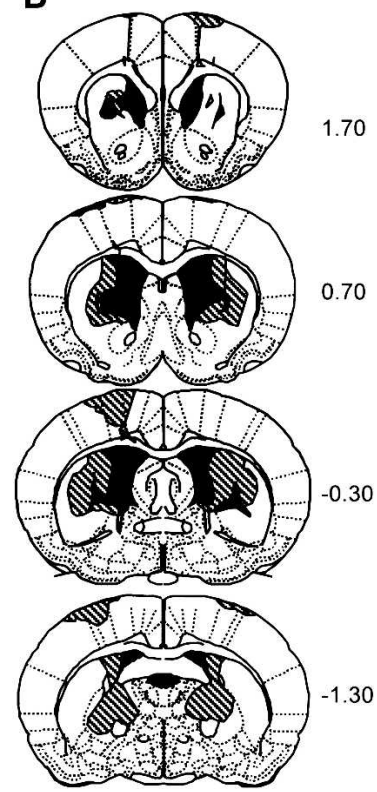

Figure 1. (A) Reconstructions of coronal sections through the hippocampus showing the smallest (black) and largest (stippled) lesion for the hippocampal lesion group. The average size of the hippocampal lesions was $85 \%$. Numbers (right) represent the distance (in millimeters) posterior to bregma. (B) Reconstructions of coronal sections through the dorsal striatum showing the smallest (black) and largest (stippled) lesion for the dorsal striatum lesion group. Numbers (right) represent the distance (in millimeters) relative to bregma. The average size of the dorsal striatum lesion was $34.6 \%$.
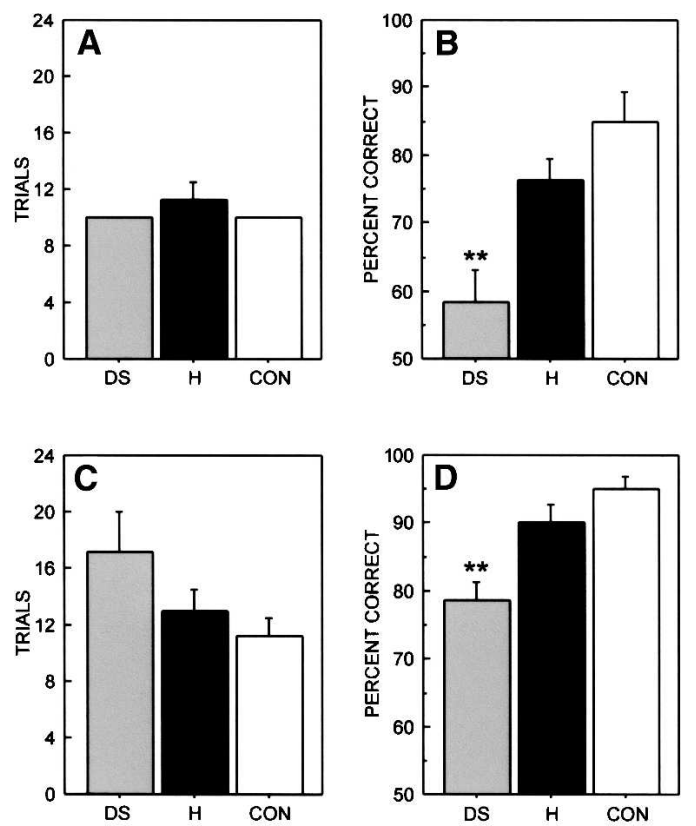

Figure 2. Odor discrimination. $(A)$ Preoperative acquisition of a twochoice odor discrimination by controls (CON, $n=6)$ and rats in the dorsal striatum (DS, $n=6)$ or hippocampal $(H, n=8)$ groups. (B) Postoperative retention of the odor discrimination 17-19 d after learning. (C) Postoperative acquisition of a new two-choice odor discrimination. (D) Retention 13-16 d after acquiring the new odor discrimination. Brackets show the standard error of the mean. (**) Impairment relative to both the control and hippocampal groups $(p<0.05)$.

(mean percent damage $=5.1 \%$; range, $0.2 \%-11.7 \%$ ). Seven animals had minor unilateral damage, and four had minor bilateral damage. In all animals, there was damage to the cortex and to the fimbria overlying the dorsal hippocampus that was associated with the placement of the syringe during surgery. There was no damage to either the amygdala or the perirhinal cortex.

\section{Dorsal striatum lesions}

Figure $1 \mathrm{~B}$ shows the smallest (black) and largest (stippled) extent of the dorsal striatum lesions. All animals sustained substantial damage to the dorsal striatum that resulted in considerable distortion of the remaining tissue. The mean percent damage was $34.6 \%$ (range, $23.4 \%-45.9 \%$ ). In most cases, the lesion extended beyond the dorsal striatum to include the cortex overlying the dorsal striatum, the dorsomedial region of the core of the nucleus accumbens, and the lateral globus pallidus, with minor encroachment into the internal capsule. In two animals, there appeared to be thinning of the lateral septal nucleus. In addition, some damage occurred to the corpus callosum, likely associated with mechanical damage caused by the radiofrequency probe. In all animals, the posterior-most region of the dorsal striatum was spared. In the rat with the largest lesion, there was minor damage to thalamic nuclei at the posterior margin of the lesion (bregma, $-1.30 \mathrm{~mm}$ ) (see Fig. 1B).

\section{Behavior}

\section{Odor discrimination}

Preoperative acquisition

Rats learned the odor discrimination quickly (Fig. 2A), and the three groups required a similar number of trials and errors to reach criterion preoperatively (dorsal striatum, DS $=10.0 \pm 0.0$ trials, $1.8 \pm 1.7$ errors; hippocampus, $\mathrm{H}=11.3 \pm 1.3$ trials, 
$2.1 \pm 0.4$ errors; Control, $\mathrm{CON}=10.0 \pm 0.0$ trials, $1.8 \pm 0.2$ errors). Analysis of the probe trials (trials 8-10) showed that the rats were discriminating based on the odor of the sand rather than by detecting the reward. All groups performed well on these trials (DS, $100 \% \pm 0.0 \% ; \mathrm{H}, 100 \% \pm 0.0 \%$; CON, $86.7 \% \pm 8.2 \%$ ).

\section{Postoperative retention}

Figure $2 \mathrm{~B}$ shows the postoperative retention scores for the odor discrimination 17-19 d after learning. An ANOVA revealed a significant effect of group $\left(F_{2,17}=10.4, p<0.01\right)$. Unpaired $t$-tests showed further that dorsal striatum lesions impaired retention of the preoperatively acquired odor discrimination relative to both the control group and the hippocampal lesion group (DS, $58.3 \% \pm 4.8 \%$ versus $\mathrm{CON}, 85 \% \pm 4.3 \% ; \mathrm{H}, 76.3 \% \pm 3.2 \%$; ts $>3.2$, ps $<0.01)$. Rats with hippocampal lesions and control rats performed similarly $(p=0.1)$.

\section{Postoperative acquisition and retention}

Figure 2C shows the number of trials needed to learn the twochoice odor discrimination postoperatively. An ANOVA revealed no significant effect of group $\left(F_{2,22}=2.3, p=0.1\right)$. Nonetheless, post hoc tests showed that rats with dorsal striatum lesions were marginally impaired at learning (DS, $17.1 \pm 2.9$ trials; CON, $11.3 \pm 1.3$ trials; $t_{13}=2.0, p=0.07$ ), and that rats with hippocampal lesions learned normally $\left(\mathrm{H}, 13.0 \pm 1.5\right.$ trials; $t_{16}=0.9$, $p=0.4$ ). Figure $2 \mathrm{D}$ shows retention scores $13-16 \mathrm{~d}$ after learning. An ANOVA revealed a significant effect of group $\left(F_{2,22}=10.6\right.$, $p<0.001)$, and unpaired $t$-tests indicated further that rats with dorsal striatum lesions were impaired on the retention test relative to both the control group and the hippocampal lesion group (DS, $78.6 \% \pm 2.6 \%$ correct; $\mathrm{H}, 90.0 \% \pm 2.3 \%$ correct; CON, $95.0 \% \pm 1.2 \%$ correct; $t \mathrm{~s}>3.0$, ps $<0.01$ ). Rats with hippocampal lesions and control rats performed similarly $(p=0.16)$. Interestingly, for the dorsal striatum group there was no difference between performance on the final 10 trials of acquisition and performance on the 10 trials of the retention test $(t=0.55$, $p=0.6$ ). Thus, the impairment exhibited by the dorsal striatum group on the retention test may be related to the poor performance of this group during initial learning.

\section{Object discrimination}

\section{Preoperative acquisition}

The three groups required a similar number of trials and errors to reach criterion preoperatively (DS, $60.0 \pm 4.7$ trials, $23.8 \pm 1.9$ errors; $\mathrm{H}, 58.0 \pm 5.5$ trials, $23.7 \pm 2.2$ errors; CON, $55.0 \pm 5.0$ trials, $21.6 \pm 1.9$ errors) (Fig. 3A).

\section{Postoperative retention}

Figure 3B shows postoperative retention scores 17-21 d after surgery. An ANOVA comparing each group's performance on postoperative retention revealed a significant effect of group $\left(F_{2,24}=8.8, p<0.01\right)$. $t$-Tests showed further that both the hippocampal and the dorsal striatum groups were impaired relative to controls (DS, $66.7 \% \pm 2.4 \%$ correct; $\mathrm{H}, 72.0 \% \pm 3.3 \%$ correct; $\mathrm{CON}, 83.8 \% \pm 2.6 \%$ correct; $t \mathrm{~s}>2.7$, $p \mathrm{~s}<0.05)$. The dorsal striatum and the hippocampal groups performed similarly $\left(t_{17}=1.3\right.$, $p=0.2)$.

Postoperative acquisition and retention of a new object pair

Figure 3C shows the number of trials needed to learn a new object pair. The three groups required a similar number of trials and errors to reach criterion (DS, $55.6 \pm 6.5$ trials, $22.9 \pm 2.8$ errors; $\mathrm{H}, 58.0 \pm 4.7$ trials, $21.6 \pm 1.2$ errors; CON, $55.0 \pm 5.0$ trials, $22.0 \pm 2.4$ errors). The results were the same when the data were based on the first four trials of each test day (Teng et al. 2000). Figure 3D shows retention scores 2 wk after learning.
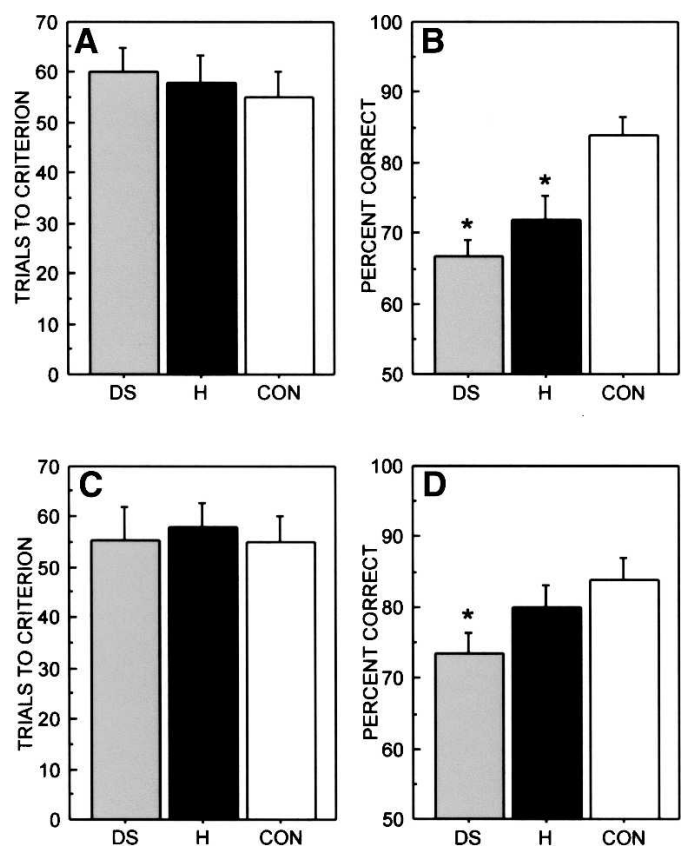

Figure 3. Object discrimination. $(A)$ Preoperative acquisition of a twochoice object discrimination by controls (CON, $n=9)$ and rats in the dorsal striatum (DS, $n=8)$ or hippocampal groups $(H, n=10)$. (B) Postoperative retention of the object discrimination 17-21 d after learning. (C) Postoperative acquisition of a new object discrimination. (D) Retention 2 wk after acquiring the new object discrimination. Brackets show the standard error of the mean. $\left(^{*}\right)$ Impairment relative to the control group $(p<0.05)$.

An ANOVA revealed a marginally significant group effect $\left(F_{2,24}=2.9, p=0.075\right)$. Unpaired $t$-tests revealed that rats with dorsal striatum lesions were impaired on the retention test relative to controls (DS, $73.3 \% \pm 2.9 \%$ correct versus CON, $83.8 \% \pm 3.2 \%$ correct; $\left.t_{15}=2.4, p=0.03\right)$. The hippocampal lesion group and the control group performed similarly $(\mathrm{H}$, $80.0 \% \pm 3.0 \%$ correct versus $\mathrm{CON}, 83.8 \% \pm 3.2 \%$ correct; $\left.t_{16}=0.8, p=0.4\right)$.

\section{Pattern discrimination}

\section{Postoperative acquisition}

Figure $4 \mathrm{~A}$ shows the number of trials needed to learn the pattern discrimination. An ANOVA revealed no effect of group $\left(F_{2,23}=0.6, p=0.6\right)$, and the three groups obtained similar scores (DS, $302.2 \pm 59.9$ trials, $136.8 \pm 30.5$ errors; H, $306.7 \pm 75.6$ trials, $134.6 \pm 36.0$ errors; CON, $220.0 \pm 39.6$ trials, $99.8 \pm 20.3$ errors; for all between-group comparisons, $t<1.11$, $p s>0.28$ ). Nevertheless, it was the case that three of the nine rats with hippocampal lesions and one rat with dorsal striatum lesions did not learn the task and were performing $\leq 60 \%$ correct after 600 training trials. These animals were given the maximal score of 600 trials and were not subsequently tested for retention. Additionally, when these animals were removed from the comparison of the number of trials to reach criterion by each group, the conclusions did not change.

Figure 4B shows retention scores 2 wk after learning. An ANOVA revealed a significant effect of group $\left(F_{2,19}=26.5\right.$, $p<0.0001)$. Further comparisons revealed that both the hippocampal and the dorsal striatum groups were impaired on the retention test relative to the control group $(\mathrm{H}, 70.0 \% \pm 2.6 \%$ correct, DS, $61.3 \% \pm 2.3 \%$ correct, CON, $81.3 \% \pm 1.3 \%$ correct; ts $>4.3, p s<0.01)$. In addition, rats with dorsal striatum lesions 

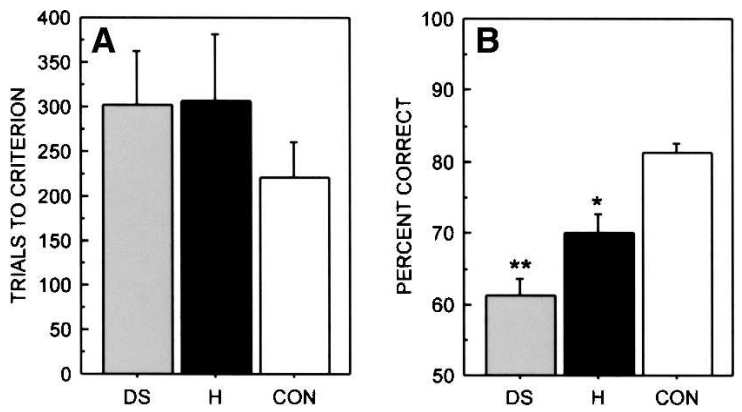

Figure 4. Pattern discrimination. $(A)$ The number of trials needed to learn a pattern discrimination by controls (CON, $n=8)$ or rats with dorsal striatum (DS, $n=9)$ or hippocampal $(\mathrm{H}, n=9)$ lesions. (B) Retention $2 \mathrm{wk}$ after learning by each group (CON, $n=8 ; \mathrm{DS}, n=8 ; \mathrm{H}, n=6)$. Brackets show the standard error of the mean. $\left(^{*}\right)$ Impairment relative to the control group $(p<0.05)$. ( $\left.{ }^{* *}\right)$ Impairment relative to both the control and hippocampal groups $(p<0.05)$.

performed more poorly than rats with hippocampal lesions $\left(t_{12}=2.5, p=0.03\right)$.

Comparison of the effect of dorsal striatum and hippocampal lesions on delayed retention of postoperatively learned odor, object, and pattern discriminations

To compare the effects of lesions across all three discrimination tasks, we calculated mean $z$ scores for each lesion group for each task (based on the performance of the controls), as well as an overall mean $z$ score for all three tasks (Fig. 5). This analysis was based on the delayed retention test that followed postoperative learning, because these data were available for all three tasks. Rats with dorsal striatum lesions performed more poorly than rats with hippocampal lesions on the odor task (DS, $z=-3.1 \pm 0.5$ versus $H, z=-0.9 \pm 0.5 ; t_{15}=3.0, p=0.01$ ) and on the pattern discrimination task (DS, $Z=-5.7 \pm 0.6$ versus $H$, $\left.z=-3.2 \pm 0.7 ; t_{12}=2.5, p=0.03\right)$. The mean $z$ scores for all three tasks indicate that, overall, the dorsal striatum lesion group performed worse than the hippocampal lesion group (DS, $z=-3.3 \pm 0.3$ versus $H, z=-1.2 \pm 0.3 ; t_{17}=5.4, p=0.001$ ), and both groups performed more poorly than the control group (ts $>4.1, p s<0.01$ ).

\section{Discussion}

Memory for two-choice discrimination tasks was severely impaired by lesions of the dorsal striatum. Damage to the dorsal striatum impaired the postoperative retention of preoperatively acquired odor and object discriminations (Figs. 2B, 3B) and also impaired delayed retention of postoperatively acquired odor, object, and pattern discriminations (Figs. 2D, 3D, 4B). Dorsal striatum lesions also marginally impaired initial learning of the odor discrimination (Fig. 2C). In contrast to these consistent and severe impairments following dorsal striatum lesions across three different types of discrimination tasks, hippocampal lesions produced a more modest effect. There was no noticeable effect on odor discriminations (Fig. 2B,D) or on delayed retention of a postoperatively acquired object discrimination (Fig. 3D). Hippocampal lesions impaired only the postoperative retention of a preoperatively acquired object discrimination (Fig. 3B), and delayed retention of a postoperatively acquired pattern discrimination (Fig. 4B). In these two cases where hippocampal lesions did impair performance, the deficit after dorsal striatum lesions was either numerically larger (Fig. 3B) or significantly larger (Fig. 4B) than after hippocampal lesions. Figure 5 summarizes the findings across all three discrimination tasks in the case of delayed retention of postoperative learning. Overall, dorsal striatum lesions produced a significantly greater deficit than hippocampal lesions.
Tasks that engage habit memory have typically been characterized as tasks that are acquired gradually over many trials through the establishment of stimulus-response associations (Mishkin et al. 1984; Packard et al. 1989). For example, in the monkey tasks such as concurrent discrimination or pattern discrimination, which are learned in several hundred trials, are impaired by caudate lesions but not by hippocampal lesions (Divac et al. 1967; Zola-Morgan and Squire 1984; Buffalo et al. 1998; Teng et al. 2000; Fernandez-Ruiz et al. 2001). Yet, tasks that are acquired rapidly by the monkey, such as easy object discriminations or trial-unique delayed nonmatching to sample, are impaired by hippocampal lesions (Teng et al. 2000; Fernandez-Ruiz et al. 2001), and, at least in the case of delayed nonmatching to sample, unpaired by caudate lesions (Fernandez-Ruiz et al. 2001). These earlier findings gave support to the idea that tasks impaired by hippocampal lesions and tasks impaired by dorsal striatum lesions differ in how fast the task can be learned.

Our study tested this idea in the rat by using three twochoice discrimination tasks (odor, object, and pattern) that differed in the number of trials required for learning (fast, intermediate, and slow). Contrary to the findings with monkeys, we found that the rate of initial learning was unrelated to whether the task was hippocampus dependent or dependent on the dorsal striatum. The most striking finding was that performance on the task learned most rapidly (the odor discrimination task) was severely impaired by dorsal striatum lesions and entirely spared by hippocampal lesions. Animals acquired the odor discrimination in only 10 trials. Yet, rats with dorsal striatum lesions were severely impaired at retaining both preoperatively acquired and postoperatively acquired odor discriminations. Furthermore, they were marginally impaired $(p=0.07)$ in acquiring the discrimination postoperatively. Together, these findings indicate that discrimination learning in the rat is primarily supported by the dorsal striatum and that rats will engage a habit-based memory system even for tasks that take only a few trials to acquire.

In the rat, the dorsal striatum is anatomically heterogeneous. It has been suggested that the dorsomedial and dorsolateral striatum are functionally distinct and that the dorsolateral striatum supports the stimulus-response learning that has traditionally been described as habit formation (Yin and Knowlton 2006). In the present study, like many studies in the rodent, we attempted to produce large lesions of the dorsal striatum that encompassed both its dorsomedial and dorsolateral aspects. Accordingly, the present study cannot speak to the issue of functional heterogeneity within the dorsal striatum, nor can it indi-

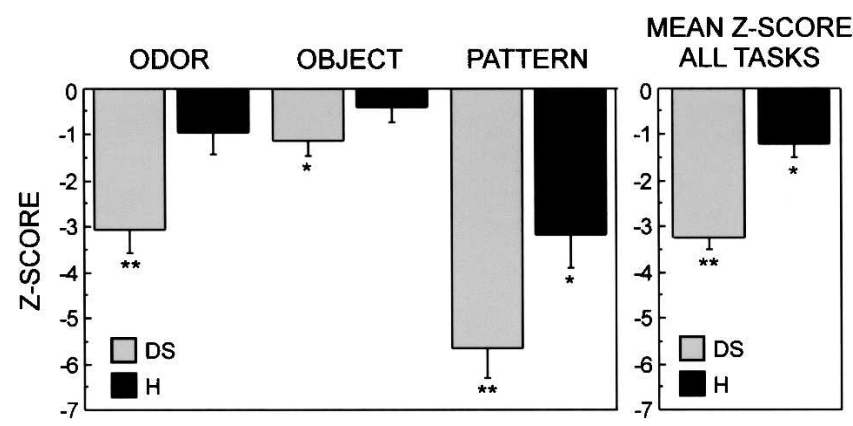

Figure 5. $\quad Z$ scores for $2-w k$ retention of postoperative learning by the hippocampal and dorsal striatum lesion groups across the three discrimination tasks (odor, object, and pattern discrimination). The $z$ scores are based on Figures 2D, 3D, and 4B. $Z$ score calculations were based on control scores, so that a mean of zero represents control performance. Brackets show the standard error of the mean. $\left({ }^{*}\right)$ Impairment relative to the control group $(p<0.05)$. $\left.{ }^{* *}\right)$ Impairment relative to both the control and hippocampal groups $(p<0.05)$. 
cate whether damage in the dorsolateral striatum was more responsible than dorsomedial damage for the impairments we observed. It is also possible that the impairment in discrimination performance associated with our dorsal striatum lesions reflects impaired habit memory (from the dorsolateral damage), together with additional impairment (from the dorsomedial damage). Further studies with smaller lesions would be useful to determine if there is a functional distinction between dorsomedial and dorsolateral striatum lesions in the case of two-choice discrimination learning and memory. In other work, dorsolateral lesions, but not dorsomedial lesions impaired acquisition of a conditional discrimination (Featherstone and McDonald 2004a,b, 2005a). Interestingly, both dorsomedial and dorsolateral striatal lesions impaired the postoperative retention of a preoperatively trained conditional discrimination (Featherstone and McDonald 2005b).

The finding that rats do not readily engage hippocampusdependent memory when performing discrimination tasks is consistent with earlier work. The situation is clearest in the case of odor discriminations. Hippocampal lesions did not impair either postoperative acquisition or retention of preoperatively acquired two-choice odor discriminations (Kaut and Bunsey 2001; Jonasson et al. 2004; Wood et al. 2004). Furthermore, retention of postoperatively acquired two-choice odor discriminations was spared following delays of $5 \mathrm{~d}$ (Kaut and Bunsey 2001) or $7 \mathrm{~d}$ (Jonasson et al. 2004).

One interesting possibility is that the hippocampus might become important for discrimination learning in the rat if the discrimination could be acquired in only one or two trials. One hallmark of hippocampus-dependent (declarative) memory in humans is that information is often acquired in a single learning episode. The rat may tend to depend on the dorsal striatum when a discrimination task requires multiple trials to learn (even as few as 10). The present findings leave open the possibility that the hippocampus might become important if the task could be learned in just one or two trials.

In the case of object discriminations, most studies have found, as we did, that acquisition is intact in rats with hippocampal lesions (Wible et al. 1992; Vnek and Rothblat 1996; Mumby et al. 1999; but see Mumby et al. 1995). We also found no impairment after hippocampal lesions in retention of a postoperatively acquired object discrimination (Fig. 3D), consistent with previous findings following a 10-d delay with twodimensional stimuli (Driscoll et al. 2005). Nevertheless, impaired retention in this condition has been reported after a 3-wk delay (Vnek and Rothblat 1996). The only impairment in object discrimination performance that we observed after hippocampal lesions was for retention of a preoperatively acquired object discrimination task. This finding has also been reported previously (Driscoll et al. 2005; but see Mumby et al. 1999). One possibility is that under ordinary conditions (with an intact brain), the hippocampus can contribute to learning and memory of object discriminations (as indicated by the impairment that we found when hippocampal lesions were made after learning) (Fig. 3B). However, when the hippocampus is damaged before learning (Fig. 3D), other brain regions, especially the dorsal striatum, can support learning and retention.

These considerations lead to the suggestion that rodents may learn object discrimination tasks differently than either monkeys or humans. Both monkeys and patients with hippocampal lesions are impaired in the acquisition and retention of simple object discriminations (Squire et al. 1988; Teng et al. 2000). In contrast, rats with hippocampal lesions are either intact or only modestly impaired. For rats, the dorsal striatum appears to be the more important structure.

One reaches a similar conclusion in the case of pattern dis- crimination. Most studies have found postoperative acquisition to be intact after hippocampal lesions in the rat (Alexander et al. 1974; Winocur 1979; Packard and McGaugh 1992; but see Olton 1972). In contrast, pattern discrimination learning has been found to be impaired after dorsal striatum lesions (Packard and McGaugh 1992). Although in our study rats with dorsal striatum lesions were numerically but not significantly worse at acquisition, they were severely impaired at retention after a delay of 14 d. Furthermore, performance on the delayed retention test following dorsal striatum lesions was significantly poorer than after hippocampal lesions.

We suggest that rats have a greater predisposition to use habit memory to solve two-choice discriminations than do monkeys or humans. In support of this idea is the finding that patients with hippocampal lesions are impaired at acquiring and retaining both object and pattern discriminations (Squire et al. 1988) and that monkeys with hippocampal lesions are impaired at acquiring simple object discriminations (Teng et al. 2000). Yet, our rats with hippocampal lesions did not exhibit these deficits. Instead, impaired performance on discrimination tasks tended to be associated with dorsal striatum lesions. Nevertheless, our suggestion is a tentative one, as monkeys and humans with selective dorsal striatum lesions have not been tested on simple object discriminations or on odor discriminations. To our knowledge, the only available evidence in this regard is that in monkeys, caudate lesions did not exacerbate the effects of hippocampal lesions on simple object discrimination learning (Teng et al. 2000). This finding is supportive of our suggestion that the dorsal striatum is less important for discrimination performance in humans and monkeys than in rats.

In summary, there were early suggestions that what determines whether a task depends on declarative memory (and the hippocampus) or on habit memory (and the dorsal striatum) is how rapidly the task is ordinarily learned. We found that the rat tends to engage habit memory regardless of the rate at which a task is learned. Strikingly, lesions of the dorsal striatum severely impaired memory for odor discriminations, despite the fact that the task could be acquired in only 10 trials. Hippocampal lesions had no effect on memory for odor discriminations. Furthermore, dorsal striatum lesions impaired retention of both object and pattern discriminations, whereas hippocampal lesions had a more modest effect. Rats appear to rely on the dorsal striatum to support memory for two-choice discrimination tasks, regardless of whether the task is learned rapidly or only gradually.

\section{Materials and Methods}

\section{Subjects}

The subjects were 47 male, Long-Evans rats weighing 300-350 g at the beginning of the study. Rats were housed individually and maintained on a 12:12-h light:dark cycle. Before surgery, rats were reduced to $80 \%$ of their free-feeding weight, and diet adjustments were subsequently introduced depending on the level of motivation exhibited during testing. Water was available ad libitum.

\section{Design}

Two different cohorts of animals were used. One cohort of animals was trained on the two-choice odor discrimination (preoperative acquisition, surgery, postoperative retention). A second cohort of animals was trained on the two-choice object discrimination task (preoperative acquisition, surgery, postoperative retention, and then postoperative acquisition and delayed retention of a new object discrimination). This group was then tested for postoperative acquisition and delayed retention of the twochoice odor discrimination, followed by postoperative acquisition and delayed retention of the pattern discrimination. For 
both groups, surgery occurred the day after reaching criterion on the preoperatively learned task.

\section{Apparatus and procedure}

\section{Two-choice odor discrimination}

Testing was conducted in the home cage in a testing room that contained a number of constant, salient visual cues (shelves, table, curtain). The stimuli consisted of $150 \mathrm{~g}$ of playground sand scented (1\% by weight) with either cinnamon or cocoa. The two odors were presented in separate glass jars $(5.5 \mathrm{~cm}$ in diameter, 7 $\mathrm{cm}$ tall), which were held firm in a plastic holder. Froot Loops cereal served as the reward.

Rats were shaped over a period of $5 \mathrm{~d}$ to dig for Froot Loops rewards in a jar of sand. Once the rats were reliably digging in the sand for reward, they were trained to discriminate between a pair of odors. On each trial, a pair of jars was presented at one end of the home cage. The odor in one jar $(\mathrm{S}+)$ was associated with a Froot Loops reward buried at least $1.5 \mathrm{~cm}$ below the surface of the sand. The jar with the other odor $(\mathrm{S}-$ ) was never associated with reward. Which odor served as the $\mathrm{S}+$ was counterbalanced across rats, and the position of the $\mathrm{S}+$ stimulus (left/right) varied pseudorandomly from trial to trial. A response was defined as any displacement of sand by either the paw or nose. Rats were allowed to self-correct if they began digging in the $S$ - cup, but the trial was recorded as incorrect. Ten trials were given each day. Trials 8-10 served as probe trials to ensure that rats were choosing based on the odors and not because they could detect the reward. On these trials, no Froot Loops were present, but a reward was placed on top of the sand when the correct odor was selected. Training continued until rats achieved a criterion of 80\% correct choices in a single session. For rats trained preoperatively, surgery occurred the day after reaching criterion on the task, and a 10trial retention test was given 17-19 d after surgery. Rats trained postoperatively were returned to the colony for the retention interval. Thirteen to $16 \mathrm{~d}$ after the completion of training, the groups were given a 10-trial retention test.

\section{Two-choice object discrimination}

Testing was conducted in the room where the odor discrimination was tested. A CD player was used to mask environmental noise. The testing apparatus was as described by Mumby et al. (1990) and Clark et al. (2001). Briefly, it was constructed from sturdy opaque Plexiglas and consisted of a $70-\mathrm{cm}$ runway that separated two identical goal areas. Access to goal areas was controlled by opaque guillotine doors. Each goal area contained two sunken food wells. Sucrose pellets ( $45 \mathrm{mg}$; Noyes Precision Food Pellets) could be delivered to the wells manually through copper tubes that led to the floor of the food wells. The food wells were separated by a small Plexiglas divider $(9 \times 9 \mathrm{~cm})$.

The stimuli for the object discrimination task consisted of two pairs of plastic objects of differing size, shape, and color. Each object was glued to a metal base and was large enough to completely cover the food wells but small enough to be easily displaced by the rat. Two copies of each stimulus pair were used, one pair located in one goal box and one pair in the other goal box. For each pair, one object always served as the rewarded $\mathrm{S}_{+}$ stimulus and the other served as the $\mathrm{S}-$ (nonrewarded) stimulus. Half the rats were trained with one object as the $\mathrm{S}+$ stimulus, and the other half were trained with that object as the $S$ - stimulus.

Pretraining began by habituating each rat to the apparatus for $20 \mathrm{~min}$ each day for $3 \mathrm{~d}$. During each habituation session, rats could find sucrose pellets in the food wells in each goal area. On each of the following $3 \mathrm{~d}$, rats learned to approach the doors and to move through them when they opened. At this stage, the rat was presented with a single object covering a food well, which could be displaced to find a food reward. Pretraining continued for a total of 6-7 d, until the rat was reliably displacing the object in order to receive a food reward.

The day after completion of pretraining, discrimination training began. For each trial, a pair of objects was presented in one of the two goal areas, such that one object covered each food well. One object of the pair (S+) was associated with a sucrose pellet reward, and the other object $(\mathrm{S}-$ ) was never associated with reward. Which object served as the S+ stimulus was counterbalanced across rats, and the position of the S+ stimulus (left/ right) varied pseudorandomly from trial to trial. Successive trials alternated between the two goal areas, so that animals did not need to be handled during testing. Twenty trials were given each day, and training continued until rats achieved a criterion of $80 \%$ correct choices on each of two consecutive sessions. Rats received surgery the day after completion of training.

Seventeen to $21 \mathrm{~d}$ after the completion of training, rats were given a 10-trial retention test. On the session following retention testing, rats were trained with a new pair of objects. About $2 \mathrm{wk}$ (14-16 d) after reaching criterion on the new object pair $(80 \%$ correct for two consecutive sessions), a final 10-trial retention test was given for this object pair.

\section{Two-choice pattern discrimination}

The room and the apparatus were the same as for object discrimination testing. The stimuli consisted of two pairs of blue wooden blocks $(5.9 \times 5.9 \times 3.0 \mathrm{~cm})$ with black and white alternating stripes ( $9 \mathrm{~mm}$ width) on the front face of each block. The black and white stripes were oriented vertically on one block of the pair and horizontally on the other block of the pair. Rats were trained as in the object discrimination task. Twenty trials were given each day until rats reached a criterion of $80 \%$ correct choices on each of two consecutive sessions or until 600 trials had been given. About 2 wk (13-14 d) after the completion of training, rats were given a 10-trial retention test.

\section{Surgery and histology}

Anesthesia was maintained throughout surgery with isoflurane gas $\left(0.8 \%-2.0 \%\right.$ isoflurane delivered in $\mathrm{O}_{2}$ at $\left.1 \mathrm{~L} / \mathrm{min}\right)$. The rat was placed in a Kopf stereotaxic instrument, and the incisor bar was adjusted until bregma was level with lambda. Bilateral excitotoxic hippocampal lesions were produced by local microinjections of ibotenate acid (IBO; Biosearch Technologies). IBO was dissolved in $0.1 \mathrm{M}$ phosphate-buffered saline to yield a solution with a concentration of $10 \mathrm{mg} / \mathrm{mL}(\mathrm{pH} 7.4)$. IBO was injected at a rate of $0.1 \mu \mathrm{L} / \mathrm{min}$ with a $10-\mu \mathrm{L}$ Hamilton syringe mounted on a stereotaxic frame and held with a Kopf Microinjector (model 5000 ). The syringe needle was lowered to the target coordinate and left in place for $1 \mathrm{~min}$ before beginning the injection. Following the injection, the syringe needle was left in place for a further 2 min to reduce the spread of IBO up the needle tract. A total of $0.51 \mu \mathrm{L}$ of IBO was injected into 18 sites within each hippocampus (for details, see Clark et al. 2001).

Dorsal striatum lesions were made with a radiofrequency electrode and generator (Radionics Model RF-4A). Preliminary work indicated that this method resulted in a better survival rate than either ibotenate acid or NMDA lesions. Lesions targeted the dorsal aspect of the caudoputamen, which is the area most frequently damaged in previous work that has studied the relationship of the dorsal striatum to learning and memory (for review, see Packard and Knowlton 2002). Lesions were made as follows: The electrode was first lowered to the target and left in place for $1 \mathrm{~min}$ before heating the tissue to $90^{\circ} \mathrm{C}$ for a period of $1 \mathrm{~min}$. The current to the electrode was then turned off, and the electrode was removed after the tip temperature fell to $41^{\circ} \mathrm{C}$ (for additional details, see Clark et al. 2000). The coordinates (in millimeters relative to bregma) for each of 10 target sites per hemisphere of the brain were as follows; $\mathrm{AP} 1.4, \mathrm{ML} \pm 2.2, \mathrm{DV}-4.2,-5.2 ; \mathrm{AP}$ 1.2, $\mathrm{ML} \pm 3.2, \mathrm{DV}-4.2,-5.2 ; \mathrm{AP} 0.0, \mathrm{ML} \pm 2.4, \mathrm{DV}-4.2$, $-5.2 ; \mathrm{AP}-0.4, \mathrm{ML} \pm 4, \mathrm{DV}-4.2,-5.2 ; \mathrm{AP}-0.9, \mathrm{ML} \pm 3.5$, DV $-4.2,-5.0$.

The procedure for the sham-operated groups was to anesthetize each rat, secure the rat in the stereotax, and then make a central incision exposing the skull. The skin overlying the skull was then approximated and sutured. At the completion of surgery, rats were allowed to recover on a heated pad, and once awake and responsive were returned to the home cage for a 2-wk recovery period. During recovery, rats were given free access to food and water and additional care as needed. Following the 
recovery period, rats were returned over a period of $5 \mathrm{~d}$ to preoperative levels of deprivation.

At completion of testing, the rats were administered an overdose of sodium pentobarbital and perfused transcardially with buffered $0.9 \% \mathrm{NaCl}$ solution followed by $10 \%$ formaldehyde solution (in $0.1 \mathrm{M}$ phosphate buffer at $\mathrm{pH}$ 7.4). The brains were then removed and cryoprotected in 20\% glycerol/10\% formaldehyde. Coronal sections $(50 \mu \mathrm{m})$ were cut with a freezing microtome, with sections starting anterior to the dorsal striatum and continuing caudally through the length of the hippocampus. Every fifth section was mounted and stained with thionin to assess the extent of the lesion. Hippocampal damage was calculated from 11 coronal sections (bregma -2.88 to $-6.8 \mathrm{~mm}$ in half-millimeter intervals). Entorhinal damage was calculated from three coronal sections (bregma -4.80 to $-6.80 \mathrm{~mm}$ in 1-mm intervals). Dorsal striatum damage was calculated from four coronal sections (bregma 1.70 to $1.30 \mathrm{~mm}$ in $1-\mathrm{mm}$ intervals).

\section{Acknowledgments}

This work was supported by the Medical Research Service of the Department of Veterans Affairs, the National Institute of Mental Health (MH24600), the Metropolitan Life Foundation, the National Science Foundation (0237053), and the James S. McDonnell Foundation. We thank Laura Entwistle and Daniel Guadarrama for assistance.

\section{References}

Alexander, G.B., Broome, B., and Means, L.W. 1974. The effects of an irrelevant intertrial task on pattern discrimination in rats with hippocampal damage. Bull. Psychon. Soc. 3: 459-461.

Bayley, P.J., Frascino, J.C., and Squire, L.R. 2005. Robust habit learning in the absence of awareness and independent of the medial temporal lobe. Nature 436: 550-553.

Buffalo, E.A., Stefanacci, L., Squire, L.R., and Zola, S.M. 1998. A reexamination of the concurrent discrimination learning task: The importance of anterior inferotemporal cortex, Area TE. Behav. Neurosci. 112: 3-14.

Bunsey, M. and Eichenbaum, H. 1995. Selective damage to the hippocampal region blocks long-term retention of a natural and nonspatial stimulus-stimulus association. Hippocampus 5: 546-556.

Clark, R.E., Zola, S.M., and Squire, L.R. 2000. Impaired recognition memory in rats after damage to the hippocampus. J. Neurosci. 20: $8853-8860$.

Devan, B.D. and White, N.M. 1999. Parallel information processing in the dorsal striatum: Relation to hippocampal function. J. Neurosci. 19: 2789-2798.

Divac, I., Rosvold, E., and Szwarcbart, M.K. 1967. Behavioral effects of selective ablation of the caudate nucleus. J. Comp. Physiol. Psychol. 63: $184-190$.

Driscoll, I., Howard, S.R., Prusky, G.T., Rudy, J.W., and Sutherland, R.J. 2005. Seahorse wins all races: Hippocampus participates in both linear and nonlinear visual discrimination learning. Behav. Brain Res. 164: $29-35$.

Featherstone, R.E. and McDonald, R.J. 2004a. Dorsal striatum and stimulus-response learning: Lesions of the dorsolateral, but not dorsomedial, striatum impair acquisition of a simple discrimination task. Behav. Brain Res. 150: 15-23.

Featherstone, R.E. and McDonald, R.J. 2004b. Dorsal striatum and stimulus-response learning: Lesions of the dorsolateral, but not dorsomedial, striatum impair acquisition of a stimulus-response-based instrumental discrimination task, while sparing conditioned place preference learning. Neuroscience 124: $23-31$.

Featherstone, R.E. and McDonald, R.J. 2005a. Lesions of the dorsolateral or dorsomedial striatum impair performance of a previously acquired simple discrimination task. Neurobiol. Learn. Mem. 84: 159-167.

Featherstone, R.E. and McDonald, R.J. 2005b. Lesions of the dorsolateral striatum impair the acquisition of a simplified

stimulus-response-dependent conditional discrimination task. Neuroscience 136: 387-395.

Fernandez-Ruiz, J., Wang, J., Aigner, T., and Mishkin, M. 2001. Visual habit formation in monkeys with neurotoxic lesions of the ventrocaudal neostriatum. Proc. Natl. Acad. Sci. 98: 4196-4201.

Hood, K.L., Postle, B.R., and Corkin, S. 1999. An evaluation of the concurrent discrimination task as a measure of habit learning: Performance of amnesic subjects. Neuropsychologia 37: 1375-1386.
Jonasson, Z., Ballantyne, J.K., and Baxter, M.G. 2004. Preserved anterograde and retrograde memory of rapidly acquired olfactory discriminations after neurotoxic hippocampal lesions. Hippocampus 14: $28-39$

Kaut, K.P. and Bunsey, M.D. 2001. The effects of lesions to the rat hippocampus or rhinal cortex on olfactory and spatial memory: Retrograde and anterograde findings. Cogn. Affect. Behav. Neurosci. 1: $270-286$

Mishkin, M., Malamut, B., and Bachevalier, J. 1984. Memories and habits: Two neural systems. In Neurobiology of learning and memory (eds. G. Lynch et al.), pp. 65-77. Guilford, New York.

Morris, R.G.M., Garrud, P., Rawlins, J.N.P., and O'Keefe, J. 1982. Place navigation impaired in rats with hippocampal lesions. Nature 297: 681-682.

Moss, M., Mahut, H., and Zola-Morgan, S. 1981. Concurrent discrimination learning of monkeys after hippocampal, entorhinal, or fornix lesions. J. Neurosci. 1: 227-240.

Mumby, D.G., Pinel, J.P.J., and Wood, E.R. 1990. Nonrecurring-items delayed nonmatching-to-sample in rats: A new paradigm for testing nonspatial working memory. Psychobiology 18: 321-326.

Mumby, D.G., Pinel, J.P.J., Kornecook, T.J., Shen, M.J., and Redila, V.A. 1995. Memory deficits following lesions of hippocampus or amygdale in rat: Assessment by an object-memory test battery. Psychobiology 23: 26-36.

Mumby, D.G., Astur, R.S., Weisend, M.P., and Sutherland, R.J. 1999. Retrograde amnesia and selective damage to the hippocampal formation: Memory for places and object discriminations. Behav. Brain Res. 106: 97-107.

Olton, D.S. 1972. Behavioral and neuroanatomical differentiation of response-suppression and response-shift mechanisms in the rat. $J$. Comp. Physiol. Psychol. 78: 450-456.

Packard, M.G. and McGaugh, J.L. 1992. Double dissociation of fornix and caudate nucleus lesion on acquisition of two water maze tasks: Further evidence for multiple memory systems. Behav. Neurosci. 106: $439-446$.

Packard, M.G. and McGaugh, J.L. 1996. Inactivation of hippocampus or caudate nucleus with lidocaine differentially affects expression of place and response learning. Neurobiol. Learn. Mem. 65: 65-72.

Packard, M.G. and Knowlton, B.J. 2002. Learning and memory functions of the basal ganglia. Annu. Rev. Neurosci. 25: 563-593.

Packard, M.G., Hirsh, R., and White, N.M. 1989. Differential effects of fornix and caudate nucleus lesions on two radial maze tasks: Evidence for multiple memory systems. J. Neurosci. 9: 1465-1472.

Poldrack, R.A. and Packard, M.G. 2003. Competition among multiple memory systems: Converging evidence from animal and human brain studies. Neuropyschologia 41: 245-251.

Schacter, D.L. and Tulving, E. 1994. Memory systems 1994. MIT Press, Cambridge, MA.

Squire, L.R. 1992. Memory and the hippocampus: A synthesis from findings with rats, monkeys, and humans. Psychol. Rev. 99: 195-231.

Squire, L.R. and Zola-Morgan, S. 1983. The neurology of memory: The case for correspondence between the findings for human and nonhuman primate. In The physiological basis of memory (ed. J.A. Deutsch), pp. 199-268. Academic Press, New York.

Squire, L.R. and Zola-Morgan, S. 1991. The medial temporal lobe memory system. Science 253: $1380-1386$.

Squire, L.R., Zola-Morgan, S., and Chen, K.S. 1988. Human amnesia and animal models of amnesia: Performance of amnesic patients on tests designed for the monkey. Behav. Neurosci. 102: 210-221.

Teng, E., Stefanacci, L., Squire, L.R., and Zola, S.M. 2000. Contrasting effects on discrimination learning following hippocampal lesions or conjoint hippocampal-caudate lesions in monkeys. J. Neurosci. 20: $3853-3863$.

Vnek, N. and Rothblat, L.A. 1996. The hippocampus and long-term object memory in the rat. J. Neurosci. 16: 2780-2787.

Wible, C.G., Shiber, J.R., and Olton, D.S. 1992. Hippocampus, fimbria-fornix, amygdale, and memory: Object discriminations in rats. Behav. Neurosci. 106: 751-761.

Winocur, G. 1979. Effects of interference on discrimination learning and recall by rats with hippocampal lesions. Physiol. Behav. 22: $339-345$.

Wood, E.R., Agster, K.M., and Eichenbaum, H. 2004. One-trial odor-reward association: A form of event memory not dependent on hippocampal formation. Behav. Neurosci. 118: 526-539.

Yin, H.H. and Knowlton, B.J. 2006. The role of the basal ganglia in habit formation. Nat. Rev. Neurosci. 7: 464-476.

Zola-Morgan, S. and Squire, L.R. 1984. Preserved learning in monkeys with medial temporal lesions: Sparing of motor and cognitive skills. J. Neurosci. 4: 1072-1085.

Received October 24, 2006; accepted in revised form January 9, 2007. 


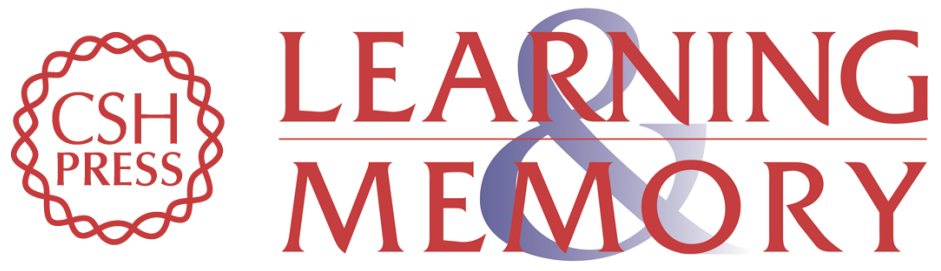

\section{Rats depend on habit memory for discrimination learning and retention}

Nicola J. Broadbent, Larry R. Squire and Robert E. Clark

Learn. Mem. 2007, 14:

Access the most recent version at doi:10.1101//m.455607

References This article cites 37 articles, 9 of which can be accessed free at:

http://learnmem.cshlp.org/content/14/3/145.full.html\#ref-list-1

License

Email Alerting Receive free email alerts when new articles cite this article - sign up in the box at the Service top right corner of the article or click here. 